K.A. van der Hucht, G. Koenigsberger \& P.R.J. Eenens, eds.

\title{
The star formation histories of two Wolf-Rayet galaxies: NGC 1741 and He2-10. Two butterflies in a collection
}

\author{
Kelsey E. Johnson \\ JILA, University of Colorado , Boulder, CO 80309-0440, USA
}

\begin{abstract}
In an effort to better understand how the properties of star formation in starburst galaxies depend on various environmental parameters, I present a comparison between two well-known WR galaxies: the interacting galaxy system NGC 1741 in the Hickson Compact Group 31, and the dwarf galaxy He2-10. The high spatial resolution of $H S T$ has allowed identification of a large number of starburst knots, or 'super star clusters' in these WR galaxies. Broad-band photometry and the latest stellar synthesis models are used to estimate the ages and masses of the super star clusters. The properties of the clusters are then used to compare and contrast the overall star-formation histories in the two WR galaxies.
\end{abstract}

\section{Introduction}

The overlap between the studies of Wolf-Rayet stars and starburst galaxies has lent itself to the investigation of some very interesting objects, and the two objects discussed here are no exception. The longterm goal, of which this work is only a small part, is to understand how the formation, evolution, and properties of star formation depend on various environmental parameters in the host galaxies. However, the parameter space is vast, and there is a great deal of work still to be done. In the meantime, I will introduce (or re-acquaint) you with the well-known WR galaxies NGC 1741 and He2-10, and overview what they may be telling us about star formation in super star clusters (SSCs).

NGC 1741 is a member of Hickson Compact Group 31, and one of the most luminous WR galaxies in Conti's (1991) catalog. In the original nomenclature of Hickson, NGC 1741 refers only to one member of the interacting pair, but because the pair is spatially connected, they are often referred to collectively as NGC 1741, a convention which I adopt in this talk. NGC 1741 is clearly an interacting system, as shown by its tidal tails, and this interaction has likely triggered the starburst event we see today. NGC 1741 is $\sim 5 \mathrm{kpc}$ across and at a distance of $51 \mathrm{Mpc}\left(H_{0}=75 \mathrm{~km} \mathrm{~s}^{-1} \mathrm{Mpc}^{-1}\right.$, Vacca \& Conti 1992). If the 30 Dor region of the LMC were inserted into this galaxy, it would only appear as one of the many SSCs.

He2-10 was the first galaxy found to exhibit WR features in its spectrum (Allen et al. 1976), and has been termed a 'prototypical' WR galaxy by Conti in his 1991 catalog. This object is relatively nearby at a distance of $9 \mathrm{Mpc}$ and is also much smaller than NGC 1741 - only $\sim 1 \mathrm{kpc}$ across. The nature and history of the starburst episode in $\mathrm{He} 2-10$ has been a subject of considerable discussion in the literature. Johansson (1987) argued that this starburst is the 


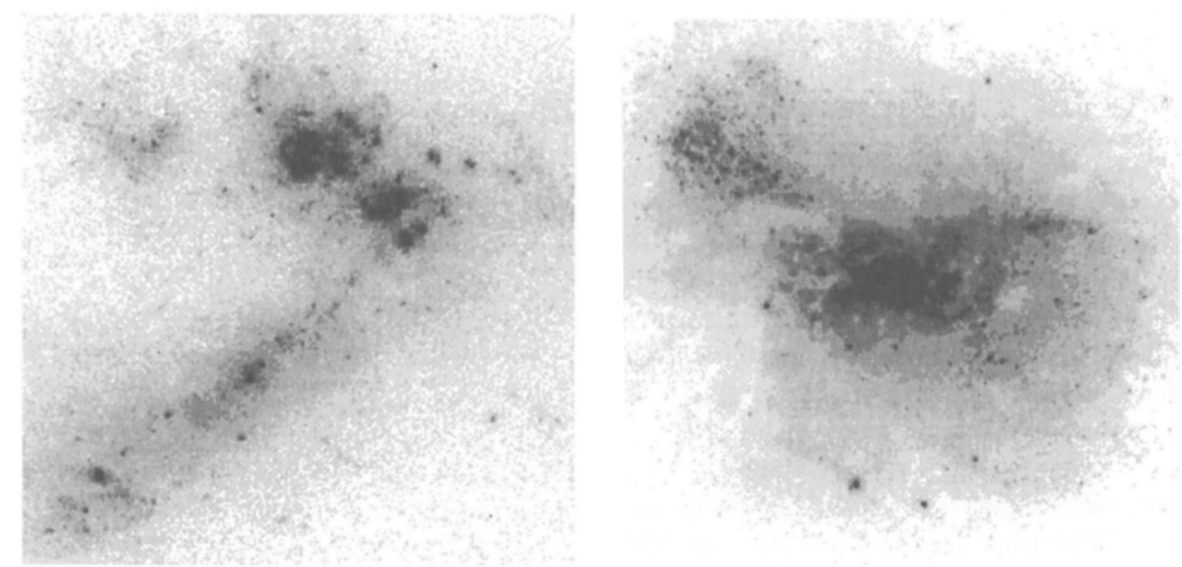

Figure 1. HST images of the two Wolf-Rayet galaxies which will be compared. Left: NGC 1741, north is toward the upper left. Right: He2-10, north is toward the top.

result of two merging dwarf irregular galaxies. However, based on the smooth elliptical envelope well fit by an $r^{1 / 4}$ law, Corbin et al. (1993) argued that the starburst is a single dwarf elliptical galaxy undergoing stochastic star formation. More recently, Kobulnicky et al. (1995) detected a molecular cloud extending to the south-east of the galaxy, which I believe to be interacting with and fueling the star formation. As Sarah Beck phrased it during this meeting, He2-10 is likely a 'little fish eating an even littler fish'.

\section{Color-magnitude diagrams}

The $V_{\mathrm{ST}} \operatorname{mag} v s . \quad V_{\mathrm{ST}}-I_{\mathrm{ST}}$ colors of the SSCs in NGC1741 and He2-10 are shown in Fig. 2. The data have not been corrected for extinction within the host galaxy, as there are likely significant variations in the amounts of gas and dust across the faces of the two galaxies. Some of the points with magnitudes fainter than $V_{\mathrm{ST}} \approx-9$ are likely to be supergiants, but it is not possible to determine specifically which ones from information so far available.

From these plots it is clear that both galaxies have undergone substantial star formation in the past $10 \mathrm{Myr}$, which is expected given that these galaxies both have a substantial population of WR stars. From the correspondence between the observed colors and the model predictions, the $V_{\mathrm{ST}}$ magnitudes of the SSCs in each galaxy are consistent with masses less than $10^{5} \mathrm{M}_{\odot}$. Although $V$ magnitudes are used to estimate the masses of the SSCs, there are a few issues of which one should be aware when using this technique. First of all, it is necessary to assume initial mass parameters (in this case a Salpeter IMF with an upper mass cutoff of $100 \mathrm{M}_{\odot}$ and a lower mass cutoff of $1 \mathrm{M}_{\odot}$ have been adopted). Also, it is likely that some of SSCs may be unresolved and contain more than one cluster, especially in the case of NGC 1741 which is further away. In addition, depending on the amount of extinction adopted, the inferred mass range could shift upward by an order of magnitude or more. 

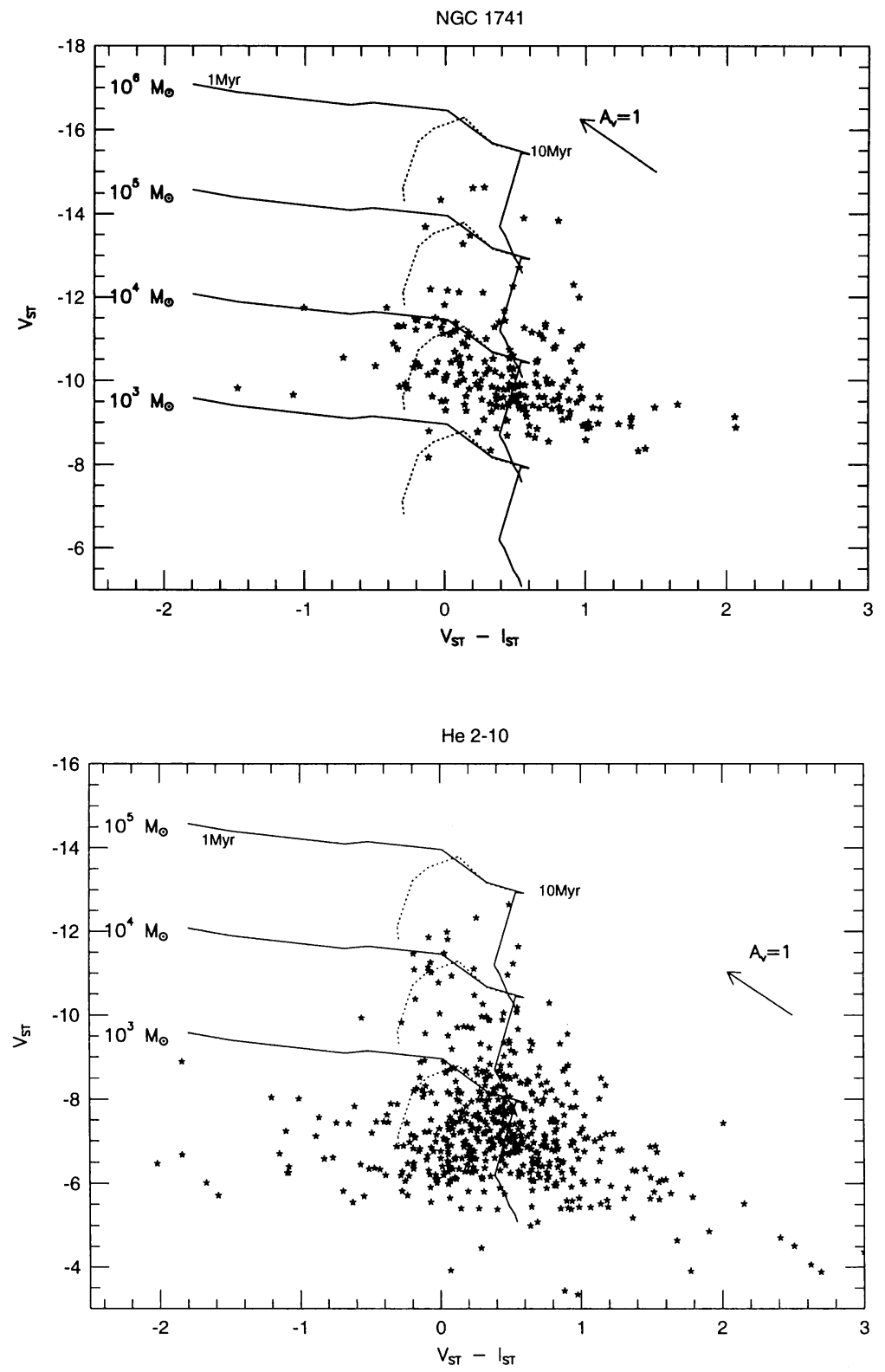

Figure 2. $V_{\mathrm{ST}}$ vs. $V_{\mathrm{ST}}-I_{\mathrm{ST}}$ diagrams for the SSCs in NGC 1741 (top) and $\mathrm{He} 2-10$ (bottom). The solid lines are models including only stellar light (Leitherer \& Heckman 1995), and the dashed lines are models also including nebular line emission (Johnson et al. 1999). The models are plotted from $1 \mathrm{Myr}$ to $100 \mathrm{Myr}$ and shown for several different masses to illustrate the dependence of the $V$ magnitude of an SSC on its mass. The data have not been corrected for reddening within the host galaxies, but the reddening vectors for an $A_{V}=1$ are shown. 


\section{Luminosity functions}

In Fig. 3 the $V_{\mathrm{ST}}$ luminosity functions for the SSCs in NGC 1741 and He2-10 are shown. Both are well fit by a power law of the form $\phi(L) \propto L^{-\alpha}$ for magnitudes brighter than the completeness limits, where $\alpha=1.9$ and 1.8 for NGC 1741 and He2-10 respectively. Luminosity functions for SSCs are generally found to have exponents in the range of $\alpha \approx 1.7$ to 2.1 (Vacca 1997; Schweizer et al. 1996; Whitmore \& Schweizer 1995).

The similarity in these slopes may provide an intriguing clue about the underlying physics in these systems. The observed slope of the luminosity function at a given wavelength is independent of reddening, and is governed by the ages of the SSCs, their stellar initial mass functions (IMFs), and their mass spectra. I will argue that in the case of starbursts of the same age, the constancy of the IMF and luminosity function slope suggests that the mass spectrum of SSCs is also constant.

The presence of a large number of WR stars in these two galaxies allows the age of the burst to be constrained a priori to $\sim 3$ to $6 \mathrm{Myr}$ using evolutionary synthesis models of stellar populations (e.g., Meynet 1995). Recent work has suggested that the slope of the high mass end of the IMF is roughly constant in a variety of local environments, from galactic $\mathrm{OB}$ associations to the closest analog of a starburst knot, R 136 in the LMC (Massey et al. 1995; Garmany 1998; Massey \& Hunter 1998). While nailing down the lowest mass end of the IMF remains one of the important goals in the field, the light from the least massive stars has little impact on the observed magnitudes of SSCs. Therefore if we take the ages of NGC 1741 and He2-10 to be the same, and if we assume there is an universal slope of the high mass end of the stellar IMF, then the strong similarity in the slopes of their luminosity functions is telling us that the mass spectrum of SSCs is also roughly constant.
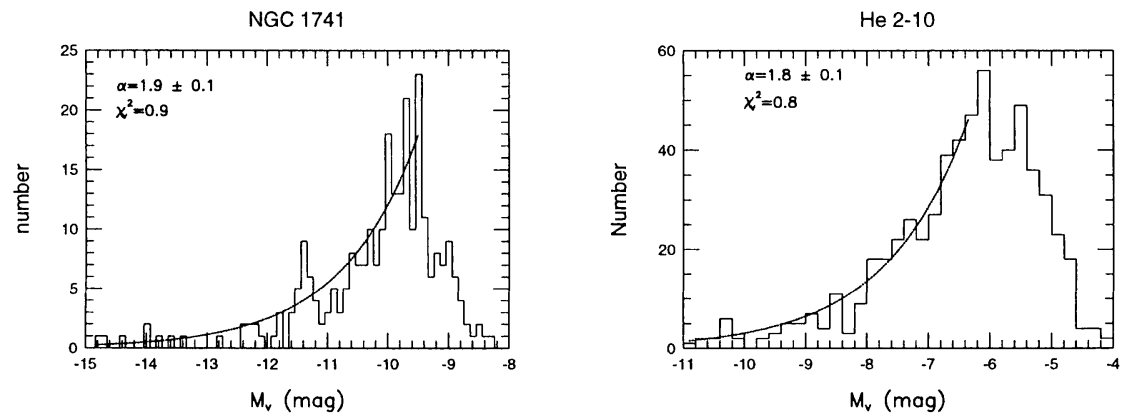

Figure 3. The $V$-band luminosity functions for the SSCs in NGC 1741 (left) and He2-10 (right). The dashed lines show the best-fit power laws of the form $\phi(L) \propto L^{-\alpha}$ for magnitudes brighter than the completeness limits. 


\section{Discussion}

In an effort to gain a better understanding of how the environmental parameters of the host galaxies affect star formation, it is useful to compare the properties of starburst galaxies, such as NGC 1741 and He2-10. Table 1 shows a side-byside comparison of some of the basic properties of these two galaxies. There are a few striking differences: NGC 1741 is relatively big, is gas poor, and has lower metallicity, whereas He2-10 is smaller, is very gas rich, and has a higher metallicity (although there is not unanimous agreement from different studies on this value).

Table 1. Basic properties of NGC 1741 and He2-10

\begin{tabular}{lcc}
\hline & NGC 1741 & He $2-10$ \\
\hline optical size & $\sim 5 \mathrm{kpc}$ & $\sim 1 \mathrm{kpc}$ \\
$M(\mathrm{HI})$ & $\sim 10^{10} \mathrm{M}_{\odot}{ }^{a}$ & $\sim 10^{9} \mathrm{M}_{\odot}{ }^{b}$ \\
$M\left(\mathrm{H}_{2}\right)$ & $\sim 3 \times 10^{8} \mathrm{M}_{\odot}{ }^{c}$ & $\sim 9 \times 10^{8} \mathrm{M}_{\odot}{ }^{b}$ \\
$M(\mathrm{HI}) / L_{B}$ & $\leq 0.2 \mathrm{M}_{\odot} / \mathrm{L}_{\odot}$ & $\geq 2 \mathrm{M}_{\odot} / \mathrm{L}_{\odot}$ \\
$Z$ & $0.25 \mathrm{Z}_{\odot}{ }^{d}$ & $0.14-0.5 \mathrm{Z}_{\odot}{ }^{d}, \geq 1 \mathrm{Z}_{\odot}{ }^{e}$ \\
typical SSC mass & $\leq 10^{4} \mathrm{M}_{\odot}$ & $\sim 10^{3} \mathrm{M}_{\odot}$ \\
interacting? & $\mathrm{YES}$ & $\mathrm{YES}$ \\
\hline
\end{tabular}

${ }^{a}$ Williams et al. $1991,{ }^{b}$ Kobulnicky et al. $1995,{ }^{c}$ Yun et al. $1997,{ }^{d}$ Vacca \& Conti 1992, ${ }^{e}$ Kobulnicky et al. 1999.

Obviously this is not a controlled experiment, as several different parameters vary between these galaxies. However, it is intriguing that despite the great differences in environmental parameters, the properties of the SSCs within these galaxies are fairly constant within our sensitivity - the typical masses of the SSCs are roughly equivalent, as are the slopes of their luminosity functions. Many more examples need to be obtained to get better statistics, and a much larger area of parameter space needs to be explored, but perhaps star formation in starburst galaxies is a more robust process than we might have dared hope.

Acknowledgments. It is a pleasure to thank Peter Conti, Bill Vacca, and Claus Leitherer for their continued discussions with me on these subjects, and all of the participants of this meeting for the lively discussions and interesting ideas to pursue in the future. Support for this work was provided by a NASA Graduate Researchers Program Fellowship, and by NASA through grant \# GO05900.01-9AA from the Space Telescope Science Institute, which is operated by the Association of Universities for Research in Astronomy, Incorporated, under NASA contract NAS5-26555. 


\section{References}

Allen, D.A., Wright, A.E., Goss, W.M. 1976, MNRAS 177, 91

Conti, P.S. 1991, ApJ 377, 115

Corbin, M.R., Korista, K.T., Vacca, W.D. 1993, AJ 105, 1313

Garmany, C.D. 1998, in: C.E. Woodward, J.M. Shull \& H.A. Thronson Jr. (eds.), Origins, ASP-CS 148, 184

Johansson, I. 1987, A\&A 182, 179

Johnson, K.E., Vacca, W.D., Leitherer, C., Conti, P.S., Lipscy, S.J. 1999, AJ in press

Kobulnicky, H.A., Dickey, J.M., Sargent, A.I., Hogg, D.E., Conti, P.S. 1995, AJ 110, 116

Kobulnicky, H.A., Kennicutt, R.C., Pizagno, J.L. 1999, ApJ 514, 544

Leitherer, C., Heckman, T.M. 1995, ApJS 96, 9

Massey, P., Hunter, D.A. 1998, ApJ 493, 180

Massey, P., Johnson, K.E., Degoia-Eastwood, K. 1995, ApJ 454, 172

Meynet, G. 1995, A\&A 298, 767

Schweizer, F., Miller, B.W., Whitmore, B.C., Fall, S.M. 1996, AJ 112, 1839

Vacca, W.D., Conti, P.S. 1992, ApJ 401, 543

Vacca, W.D. 1997, in: W.H. Waller, M.N. Fanelli, J.E. Hollis \& A.C. Danks (eds.), The Ultraviolet Universe at Low and High Redshift: Probing the Progress of Galaxy Evolution, AIP Conf. Proc. 408, 21.

Whitmore, B.C., Schweizer, F. 1995, AJ 109, 960

Yun, M.S., Verdes-Montenegro, L., Del Olmo, A., Perea, J. 1997, ApJ 475, L21

\section{Discussion}

Lehnert: Your SSC-luminosity functions are very interesting, but how did you separate low-luminosity SSCs from luminous high-mass stars?

Johnson: In fact, I can't separate them. With broad band colors, it's impossible to distinguish faint and/or reddened SSCs from supergiants. However, as we push the completeness limits back, I think it will be interesting to see how the luminosity function changes, as more and more stellar contamination will be visible.

Massey: If IC 10 were further away, it might be called a WR galaxy. But here its 'starburst' is not happening by way of SSCs, but rather by a galaxy-wide high SF-rate. Do you have any idea yet what the statistics show? How many of the WR galaxies are doing it via SSCs, and how many are doing it via the IC 10 route?

Johnson: This is something interesting, which $I$, think we need to investigate. So far, most (all?) 'starbursts' we have observed appear to be composed of SSCs, however, not all have been observed at sufficient resolution, and I also have to wonder if there is a selection effect. Some other things to consider are the interaction (or non-interaction) history of the galaxy, as well as the Jeans mass under different conditions.

Terlevich: Can you comment on the one-colour abundance you listed in your transparancy for He2-10?

Johnson: Yes, the metalicity for He2-10 has been updated by Kobulnicky et al. to an approximately solar value. This is rather large, but not unheard off, and also Contini's results (these Proceedings) are consistent with this new value. For further information I refer you to Chip Kobulnicky's work. 